\title{
Testing of the dwarf galaxy content and the evolutionary status of nearby groups of galaxies
}

\author{
J. Vennik ${ }^{1, \star}$ and $\mathbf{U}$. Hopp ${ }^{2,3}$ \\ 1 Tartu Observatory, 61602 Tõravere, Tartumaa, Estonia \\ 2 Universitäts-Sternwarte München, Scheiner Str. 1, D 81679 München, Germany \\ 3 Max-Planck-Institut für Extraterrestrische Physik,D 85741 Garching, Germany
}

The dates of receipt and acceptance should be inserted later

\begin{abstract}
Key words galaxies: dwarf - galaxies: distances and redshifts - galaxies: clusters: individual (IC 65 group, NGC 6962 group, NGC 5005/5033 group)

We carried out visual and parametric searches for dwarf galaxies in five loose groups of galaxies. Follow-up spectroscopy with the HET has shown a 50\% success rate of morphological selection. The evolutionary status of the studied groups differs: while the NGC 6962 group has a partially relaxed core, surrounded by an infall region, the NGC 5005/5033 group and the IC 65 group, which consist only of late-type galaxies, are probably still assembling.
\end{abstract}

(C) 2006 WILEY-VCH Verlag GmbH \& Co. KGaA, Weinheim

\section{Introduction}

Groups of galaxies may represent sites for a preprocessing stage of cluster galaxies through some varieties of gravitational (tidal) and hydrodynamical mechanisms acting in groups. Dwarf galaxies are expected to reflect the environmental influence most prominently. We have selected dwarf galaxy candidates in a sample of nearby loose groups of galaxies, basing on morphological criteria including surface photometry. The main aim of our project is to investigate the impact of the group environment on the evolution of the dwarfs as well as on the evolution of the major group members due to dwarf galaxy accretion. Here we report the preliminary results obtained for three groups around the principal galaxies IC 65, NGC 6962, and NGC 5005/5033, respectively, using the archival data from the SDSS, complemented with our own imaging studies at Calar Alto, and spectroscopy with the Hobby-Eberly Telescope (HET).

\section{Candidate selection and membership confirmation progress report}

\subsection{Candidate selection on the DPOSS and with our own imaging data (IC 65 group)}

We carried out a parametric search for new dwarf galaxies in the area of the IC 65 group with SExtractor software on the DPOSS frames. For the preliminary classification of detected galaxies we used (a) the Binggeli's (1994) empirical relation between the central surface brightness (SB) and absolute magnitude, and (b) the empirical light concentration parameter (Trentham et al. 2001), versus SExtracted colour

\footnotetext{
^ e-mail: vennik@aai.ee
}

index. As result, we have selected four LSB irregular galaxies with lowest light concentration and the bluest colour $(0.45<B-R<1.05)$. We have obtained CCD $B, R$ and $I$ imaging data with the Calar Alto $1.23 \mathrm{~m}$ telescope and performed detailed surface photometry of all secure and possible members of the IC 65 group (Vennik \& Hopp 2008). The final classification of the new candidate galaxies has been carried out according to their SB (LSB, HSB), morphology (irregular, regular) and colour (blue, red).

\subsection{Candidate selection with the SDSS data (NGC 6962 and NGC 5005/5033 groups)}

The area of these two groups is covered by the Sloan Survey and we have searched for new dwarf member candidates of these groups using the homogeneous imaging data of the SDSS. We have extracted the list of the dwarf galaxy candidates from the PhotoObjAll catalog using the selection criteria as follows: the effective SB $\mu_{\mathrm{eff}}>22.0 \mathrm{~g} \mathrm{arcsec}-2$; light concentration $C=$ petroR $50 /$ petroR $90>0.4$; size iso $A>15$ arcsec. All pre-selected dwarf candidates have been visually inspected on the SDSS frames and final classification has been made on their morphological and colour grounds.

For NGC 6962 group of galaxies, we retrieved 29 probable (and 44 possible) members of the group within $1 \mathrm{Mpc}$ around the parent galaxy NGC 6962. The comparison of angular 2D correlation properties of true (confirmed by redshift) and candidate members of the NGC 6962 group shows that the ensemble of probable members may consist a mix of $\sim 55 \%$ of true members and $\sim 45 \%$ of randomly distributed "field" objects (Vennik \& Tago 2007).

For the NGC 5005/5033 group of galaxies, our survey resulted into 5 probable (and 8 possible) dwarf galaxies within $1.5 \mathrm{Mpc}$ centered on the two major group members. 


\subsection{HET spectroscopy - candidate confirmation progress report}

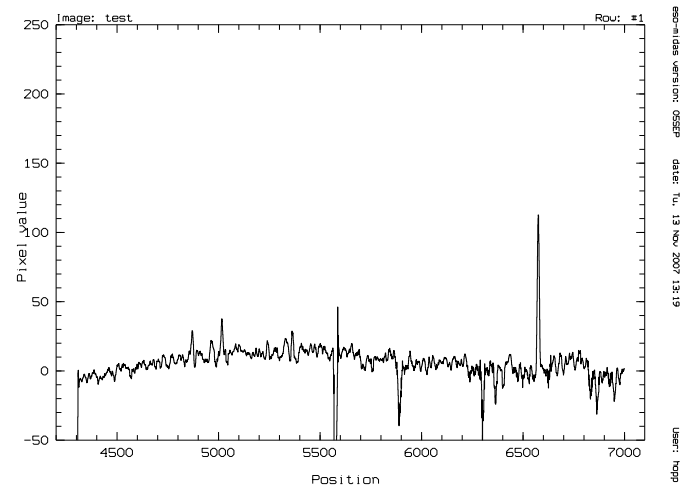

Fig. 1 HET LRIS long slit spectrum of a new dwarf member of the IC 65 group showing the typical emission lines of HII galaxies (and night sky residuals).

Follow-up spectroscopy of the selected dwarf galaxy candidates has been carried out with the HET low resolution spectrograph $\left(2^{\prime \prime} \times 4^{\prime}\right.$ long slit, Grism with $5 \AA$ or $2 \AA$ per pixel, in the range of 430 to $800 \mathrm{~nm}$ ), with aim to obtain redshifts and SED classification (Fig. 11). During several observing runs in 2007 and 200819 redshifts were obtained towards five groups with following results: 8 confirmed new members, 2 confirmed background objects, 9 member candidates rejected (either background or foreground). Among the four member candidates of the IC 65 group two are true dwarf members of this group and the other two are dwarf galaxies but members of the newly identified foreground group around the NGC 278 (Fig. 2). All four new member candidates of the NGC 6962 group, observed with the HET, are confirmed as true members of this group.

Thus, in total, the morphological classification has a success rate of $52 \%$ according to the spectroscopic follow-up. Almost all of the confirmed dwarf members are of late morphological type and show emission lines pointing to ongoing star formation activity.

\section{The NGC 6962 group}

The NGC 6962 group is a dense group at the distance of $56 \mathrm{Mpc}$. It is well isolated, both in the sky and in velocityspace. There are 38 galaxies with concordant redshifts within $r_{0}=2.6 \mathrm{Mpc}$ (zero-velocity radius) around the parent galaxy, listed in the SDSS and/or in the NED. By now, four new member candidates have been confirmed by the HET spectroscopy. The group has well-defined core - halo structure, with the core (or second turn-around) radius $r_{2 t} \simeq$ $0.73 \mathrm{Mpc}$ with 26 secure members, and halo (or infall region) extending to $r_{0}=2.6 \mathrm{Mpc}$. The group kinematics is characterized by the bi-modal velocity histogram (Fig. 3),
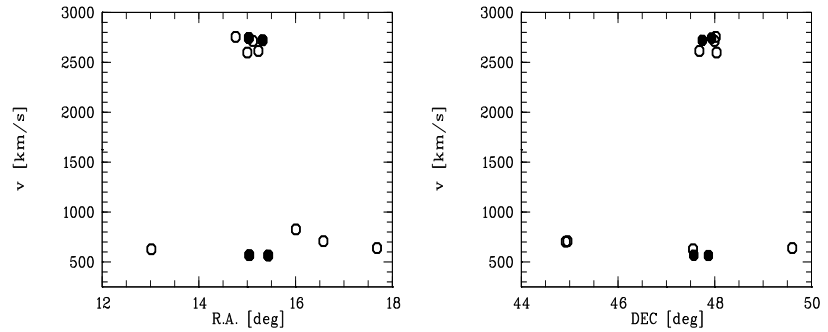

Fig. 2 Redshift distribution towards the IC 65 group: filled symbols - new redshifts measured with the HET; open symbols - redshifts obtained from the NED. The IC 65 group with two new dwarf members, confirmed by HET, has a mean heliocentric redshift $2670 \mathrm{~km} / \mathrm{s}$. The other two newly detected dwarf galaxies with $v \sim 600 \mathrm{~km} / \mathrm{s}$ are probably members of the NGC 278 group of galaxies.

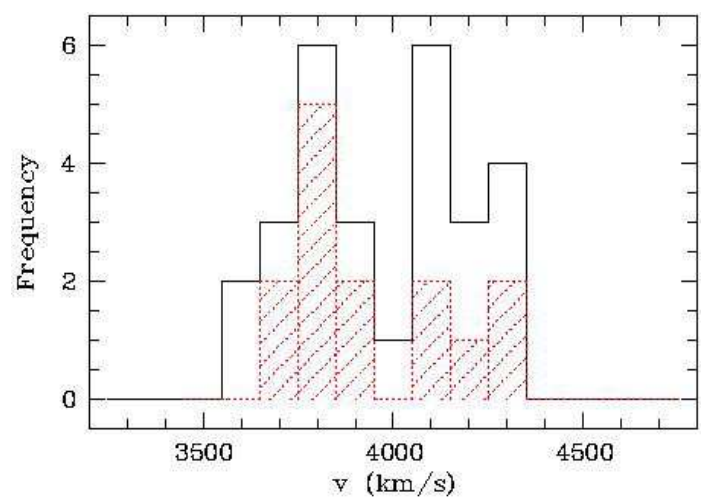

Fig. 3 The NGC 6962 group velocity distribution is bimodal with peaks at $3765 \pm 25 \mathrm{~km} / \mathrm{s}$ and $4171 \pm 27 \mathrm{~km} / \mathrm{s}$. Both velocity-subgroups almost overlap in the sky and contain nearly equal fraction of early-type (shaded histogram) and late-type galaxies.

and by several congregations of big bubbles in the DresslerShectman (1988) DS-test, which are indicative of kinematically distinct subgroups (Fig. 4). The NGC 6962 group shows a classical morphology-density (and spectral-density) correlation with early/passive types (E, E+ pec, S0) mostly in the core, and late/star-forming types in the halo.

The group luminosity function is essentially flat in the range of $-21 \leq M_{g} \leq-16$.

Evolutionary status of the NGC 6962 group: evidence of segregation, declining velocity dispersion profile, and relatively short crossing time $\left(t_{\mathrm{c}} H_{0} \simeq 0.14\right)$ of the core may be indicative of a partially relaxed group core. According to the ROSAT Survey it is an X-ray dark group, i.e. the intragroup gas, if stripped in early stages of the group evolution from spiral galaxies by mutual interactions, is still in a relatively cold stage. The disturbed $\mathrm{E}+$ pec galaxies may be late merger remnants. 


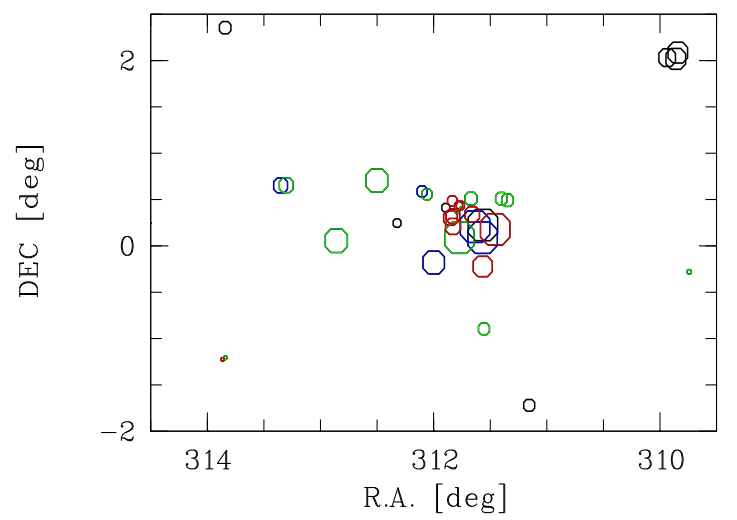

Fig. 4 Distribution of the NGC 6962 group members within $\sim 3 \mathrm{Mpc}$ around the parent galaxy (and are colourcoded blue-green-red according to their decreasing current star-forming activity, classified by us as in Tully \& Trentham (2008)). The bubble sizes are scaled according to the Dressler-Shectman (1988) DS-test for substructure - isolated congregation of large bubbles is indicative of a kinematically distinct subgroup.

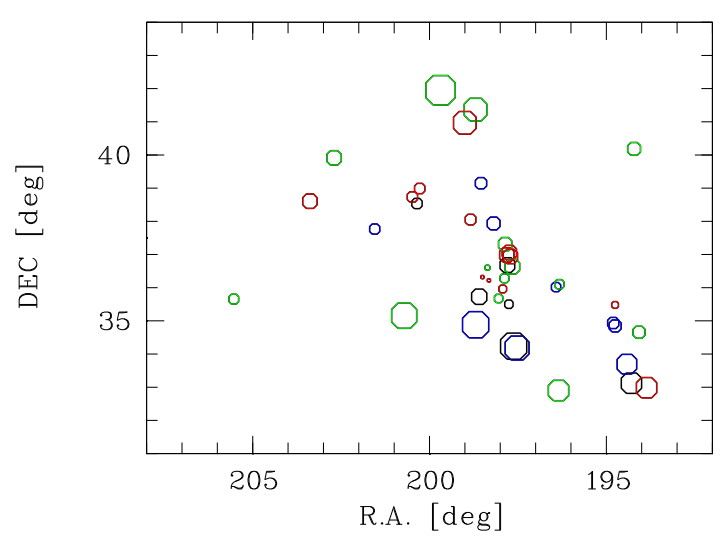

Fig. 5 The distribution of the NGC 5005/5033 group members within $\sim 1.5 \mathrm{Mpc}$ around the group barycenter. The bubble sizes are scaled according to the DS-test (as in the Fig. 4).

\section{The NGC 5005/5033 group}

This is a moderately isolated loose aggregation of 41 galaxies (SDSS \& NED) within 6 degrees $(\sim 1.5 \mathrm{Mpc})$ around the group barycenter, at the distance of $13.7 \mathrm{Mpc}$. Two new dwarf members were confirmed by our HET spectroscopy. The group consists of only late-type galaxies (later than $\mathrm{Sab}$, and is dominated by a wide pair of luminous spirals, namely NGC $5005\left(M_{B}=-21.4\right)$ and NGC 5033
$\left(M_{B}=-20.8\right)$. The group includes several kinematically distinct subgroups of faint, actively star forming galaxies (e.g. around UGC 8246 and UGC 8365), possibly falling to the group along the filament(s) (compare Fig. 5). The group velocity dispersion profile is rising with increasing radius.

Evolutionary status: the preliminary analysis let us suppose that this group is probably still assembling and star-formation in gas-rich late-type galaxies may be driven by mutual interactions, particularly in kinematically distinct subgroups.

\section{Summary}

For a sample of nearby loose groups, we complemented the member ship lists with new dwarf galaxies where the group membership has been confirmed by HET spectroscopy. The preliminary analysis of the NGC 6962 and NGC 5005/5033 groups show, that these two groups differ in their spatial structure, kinematics and morphological/spectral content, which possibly indicate their different evolutionary status. The IC 65 group has been analyzed in more detail previously (Vennik \& Hopp 2007, 2008), and the evolutionary status of this group can be summarized being at a relatively early stage of its collapse.

Acknowledgements. The research of JV has been supported by the Estonian Science Foundation grants 6106 and 7765. We are grateful to the organizers of JENAM2008. This study has made use of the NASA/IPAC Extragalactic Database (NED), the STScI Digitized Second Palomar Obsrvatory Sky Survey (DPOSS), and the Sloan Digital Sky Survey (SDSS). The Hobby-Eberly Telescope (HET) is a joint project of the University of Texas at Austin, the Pennsylvania State University, Stanford University, LudwigMaximilians-Universität München, and Georg-August-Universität Göttingen. The HET is named in honor of its principal benefactors, William P. Hobby and Robert E. Eberly.

\section{References}

Binggeli, B.: 1994, in Panchromatic View of Galaxies. Their Evolutionary Puzzle, ed. G. Hensler, C. Theis \& J.S. Gallagher (Gif-sur-Yvette: Edition Frontiers), 173

Dressler, A., Shectman, S.A.: 1988, AJ 95, 985

Trentham, N., Tully, R.B., Verheijen, M.A.W.: 2001, MNRAS 325, 385

Tully, R.B., Trentham, N.: 2008, AJ 135, 1488

Vennik, J., Tago, E.: 2007, in Groups of Galaxies in the Nearby Universe, ed. I. Saviane, V. Ivanov, \& J. Borissova, ESO Astrophysics Symposia, 119

Vennik, J., Hopp, U.: 2007, in Stellar Populations as Building Blocks of Galaxies, ed. A. Vazdekis, \& R.F. Peletier (Cambridge University Press), Proc. IAU Symp., 241, 525

Vennik, J., Hopp, U.: 2008, A\&A 481, 79 